\title{
Editorial: The Importance of Olfaction in Intra- and Interspecific Communication
}

\author{
Luisa $\mathrm{Amo}^{1 *}$ and Francesco Bonadonna ${ }^{2}$ \\ ${ }^{1}$ Departamento de Ecología Evolutiva, Museo Nacional de Ciencias Naturales (Consejo Superior de Investigaciones \\ Científicas), Madrid, Spain, ${ }^{2}$ CEFE UMR 5175, Centre National de la Recherche Scientifique - Université de Montpellier - \\ Université Paul-Valéry Montpellier - EPHE, Montpellier, France
}

Keywords: chemical communication, olfaction, behavioral ecology, chemical ecology, animal behaivor

\section{Editorial on the Research Topic}

\section{The Importance of Olfaction in Intra- and Interspecific Communication}

OPEN ACCESS

Edited and reviewed by: Jordi Figuerola, Estación Biológica de Doñana (EBD),

Spain

*Correspondence: Luisa Amo

luisa.amo@mncn.csic.es

Specialty section:

This article was submitted to Behavioral and Evolutionary Ecology,

a section of the journal

Frontiers in Ecology and Evolution

Received: 25 April 2018 Accepted: 08 May 2018

Published: 25 May 2018

Citation:

Amo L and Bonadonna F (2018) Editorial: The Importance of Olfaction

in Intra- and Interspecific

Communication.

Front. Ecol. Evol. 6:71.

doi: 10.3389/fevo.2018.00071
Chemical cues are possibly the most ancient mechanism used by organisms for communication and to assess their environment. Their use has been extensively studied in different taxa, including unicellular organisms, plants, invertebrates, and, to a lesser extent, in vertebrates (Wyatt, 2014). Olfaction, in a broad sense, is one of these chemical cues. Semiochemicals are substances, emitted by organisms, carrying a message used in communication. They can range from a single compound that induce a fixed response, such as pheromones released by females to attract males, to complex mixtures of chemical compounds that provide information about the sender to which conspecifics may or may not react depending on their motivation or physiological state (Wyatt, 2014). Several species, from insects to mammals, can share similar semiochemicals, as the case of the pheromone recently defined ELEMOTH (Berenbaum, 2016). Alternatively, they can have a species-specific blend of compounds. Semiochemicals under genetic control provide information about species, gender and kin recognition, genetic compatibility, and genetic qualities (Wyatt, 2014). For example, they may signal Major Histocompatibility Complex polymorphism participating in mate choice in different taxa (Milinski, 2006). Semiochemicals may also be influenced by the environment (e.g., diet, hormone levels, parasite load, etc.) and may broadcast health and/or emotional status (Wyatt, 2014). All this information is often used in mate choice to achieve, for instance, reproductive isolation, inbreeding avoidance, and choice of a compatible mate (Johansson and Jones, 2007). Schneeberger et al. working in a free-ranging mammal with high social complexity, the greater sac-winged bat (Saccopteryx bilineata), shows here that wing-sac liquids contains a chemical fingerprint related to the age of males, suggesting that females may use these secretions to assess whether a potential mate has reached sexual maturity. Individual and/or kin recognition may also help animals to discriminate between their own offspring and those of intraspecific parasites. Yet, social parasites can escape host detection using chemical deception in classical "arms race" (Lorenzi, 2006). Lorenzi et al. show here that the chemical signature of Polistes biglumis wasps is similar between those queens behaving as intraspecific social parasites and those that found a colony, but only in those populations where queens do not produce workers. They suggest that the presence of workers in other populations increases the selection pressure that host workers impose on the chemical signature of intraspecific social parasites.

It is not still clear how the chemical signature, the scent of an individual, is achieved. It has been hypothesized that scent may be mediated by the transformation of primary chemical compounds produced by animals. For example, in the case of birds, it has been suggested that the microbiome on feathers and/or in the uropygial gland (the most important secretory gland in birds) 
may modify the compounds present in the secretions and produce the individual odor (Mardon et al., 2011). Whittaker et al. show here the relative contributions of environments and genetics in shaping dark-eyed juncos' (Junco hyemalis) symbiotic bacterial communities and investigated whether these bacterial communities underlie juncos' chemical signaling behavior. Their results show that relatedness did not noticeably affect bacterial or volatile profiles of birds, whilst shared environments were more influential. This study points out the efforts of researchers in trying to disentangle the role of olfaction in avian life histories, a sensory mode that was, until recently, neglected. And this lack of knowledge on birds is reviewed by Krause et al. They frankly emphasize that olfaction should not be disregarded even if we do not have sufficient information about the olfactory capabilities of the bird species in question, as it may have an important role in many aspects of their biology.

Semiochemicals of conspecifics are also known to influence the behavior and physiology of receivers. D'Ettorre et al. here present the results on linguistic processing of exposing adult humans to three different androstene compounds. For example, women significantly slowed down the reaction time to competitive words after being exposed to androstenone.

The semiochemical world where animals live is more significant and complex than we think. Since we mostly consider what we believe to sense better, and we wrongly believe that olfaction is a lesser sense in humans, olfaction is often disregarded. However, the olfactory world should be taken into account, not only when trying to develop programs of reproduction for endangered species or to improve the

\section{REFERENCES}

Berenbaum, M. R. (2016). "Are mammals just furry bugs with fewer legs? Convergences in mammalian and insect chemical ecology," in Chemical Signals in Vertebrates, Vol. XIII, eds by B. A. Schulte, T. E. Goodwin and M. H. Ferkin (Heidelberg: Springer), 3-10.

Johansson, B. G., and Jones, T. M. (2007). The role of chemical communication in mate choice. Biol. Rev. 82, 265-289. doi: 10.1111/j.1469-185X. 2007.00009.x

Lorenzi, M. C. (2006). The result of an arms race: the chemical strategies of Polistes social parasites. Ann. Zool. Fenn. 43, 550-563.

Mardon, J., Saunders, S. M., and Bonadonna, F. (2011). From preen secretions to plumage: the chemical trajectory of blue petrels' Halobaena caerulea social scent. J. Avian Biol. 42, 29-38. doi: 10.1111/j.1600-048X. 2010.05113.x productivity of farm animals, but also when considering other aspects of wildlife management. Jones et al. explore here how predator scents used as a pest control can influence all trophic levels, promoting landscapes of fear that influence spatial and temporal patch use in target animals. They illustrate how the application of their conceptual model can focus future research to enhance the use of predator scent-based deterrents in conservation and management.

We hope that the contributions presented in this Research Topic will provide state of the art examples of the role of olfaction in animal behavior and promote further research in this framework.

\section{AUTHOR CONTRIBUTIONS}

LA and FB came up with the topic idea, contributed to its description and to the overall organization of the special topic. All editors contributed to the overseeing of reviews for this special issue and wrote the cover editorial.

\section{FUNDING}

LA was supported by the Ramón y Cajal programme and the MINECO project CGL2014-58890-P.

\section{ACKNOWLEDGMENTS}

We wish to thank all the contributing authors for their efforts. We also want to thank Dr. Jessica Graham for English revision.

Milinski, M. (2006). The major histocompatibility complex, sexual selection, and mate choice. Ann. Rev. Ecol. Evol. Syst. 37, 159-186. doi: 10.1146/annurev.ecolsys.37.091305.110242

Wyatt, T. D. (2014). Pheromones and Animal Behaviour. Cambridge: Cambridge University Press.

Conflict of Interest Statement: The authors declare that the research was conducted in the absence of any commercial or financial relationships that could be construed as a potential conflict of interest.

Copyright (C) 2018 Amo and Bonadonna. This is an open-access article distributed under the terms of the Creative Commons Attribution License (CC BY). The use, distribution or reproduction in other forums is permitted, provided the original author(s) and the copyright owner are credited and that the original publication in this journal is cited, in accordance with accepted academic practice. No use, distribution or reproduction is permitted which does not comply with these terms. 